\title{
IceAct: Imaging Air Cherenkov Telescopes with SiPMs at the South Pole for IceCube-Gen2
}

\author{
The IceCube-Gen2 Collaboration \\ $\dagger$ http://icecube.wisc.edu/collaboration/authors/icrc17_gen2 \\ E-mail: jauffenbeicecube.wisc.edu
}

IceCube-Gen2 is planned to extend the IceCube Neutrino Observatory at the geographic South Pole. For neutrino astronomy, a large sample of well-reconstructed astrophysical neutrinos with very low background is essential. The main background for this signal consists of muons and neutrinos, which are produced in cosmic-ray air showers in the Earth's atmosphere. The coincident detection of these air showers by the surface detector IceTop has already proven the concept of vetoing atmospheric neutrinos and muons in a limited field of view centered around the South Pole. This motivates a large extension of IceTop to detect cosmic rays more efficiently over a large field of view. As part of these extension plans, small imaging air Cherenkov telescopes based on SiPM cameras and optimized for harsh environments, referred to as IceAct are considered. Compared to IceTop stations, these telescopes may be an efficient way to lower the detection threshold for air showers at the cost of a lower duty cycle. In addition small ACTs in combination with IceTop and IceCube can improve the capabilities to measure the composition of the CR spectrum starting at 2 $\mathrm{PeV}$. We will present the progress and future plans of the IceAct project including first coincident data of an IceAct prototype with IceCube.

Corresponding author: Jan Auffenberg*1

${ }^{1}$ RWTH Aachen University

35th International Cosmic Ray Conference - ICRC2017

10-20 July, 2017

Bexco, Busan, Korea

${ }^{*}$ Speaker. 


\section{Introduction}

IceCube [1] has measured an astrophysical neutrino flux with very high significance but no evidence for a point source has been found $[2,3]$ so far. In the region above $100 \mathrm{TeV}$ primary neutrino energy the measured diffuse flux is significantly above the background of cosmic-ray induced neutrinos and muons. One of the main tasks of an extended IceCube detector, IceCube-Gen2, will be the detection and high-quality reconstruction of a sufficient number of astrophysical neutrino events for astronomical observations and the measurement of the corresponding neutrino spectrum with high precision (see also [4, 5]).

The main backgrounds for extraterrestrial neutrino detection are

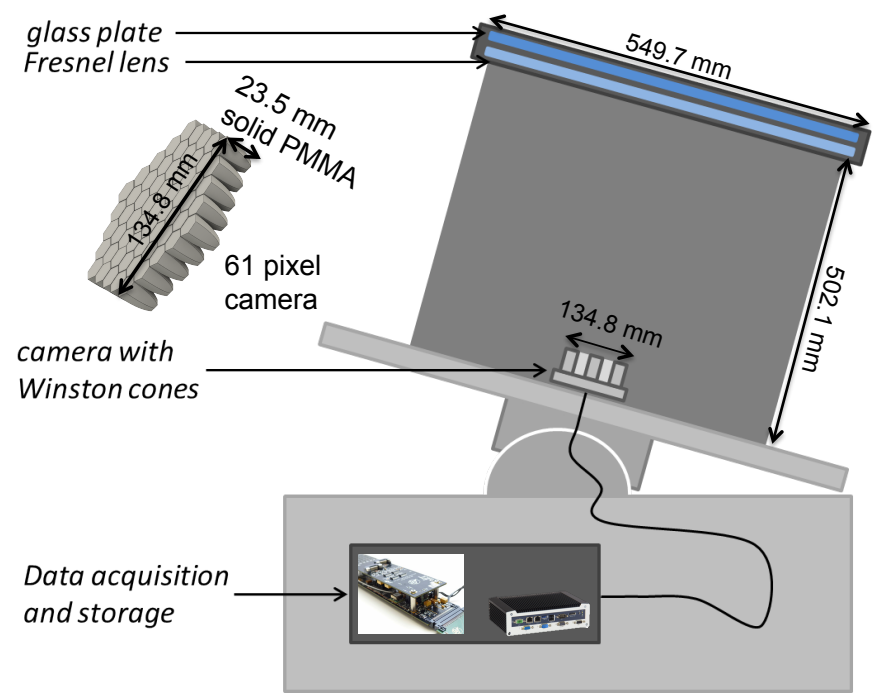

Figure 1: Drawing of the IceAct telescope prototype with a 61 pixel camera. A similar IceAct demonstrator equipped with a 7 pixel camera was deployed at the South Pole on the roof of the counting house of the IceCube neutrino observatory.

cosmic-ray induced particles. One way to suppress these backgrounds in IceCube is to look for particles that traversed the whole Earth, leaving only neutrinos - astrophysical and atmospheric. In the southern sky, the cosmic-ray induced muon background is dominant because high-energy muons $(>400 \mathrm{GeV})$ are not absorbed within at least $1.5 \mathrm{~km}$ overburden of ice above the detector. However, cosmic-ray induced air showers can be detected with dedicated air shower arrays at the surface [6]. Such a surface detector can be used to veto the cosmic-ray muons reaching the deep in-ice detector. To some extent, such a veto detector is able to select and suppress even the atmospheric neutrino background as they are also accompanied by air showers on the surface. In fact, IceTop is already used today in several analyses to suppress the atmospheric background and increase the sensitivity of IceCube for astrophysical neutrinos in the southern sky [7, 8]. The properties of IceTop as a veto motivate further studies of a specialized detector to very efficiently detect cosmic-ray muons already at the surface. This offers the possibility to select neutrinos that interacted anywhere in the entire ice sheet above the IceCube in-ice detector as astrophysical neutrino candidates. Of special interest are neutrinos from directions of possible neutrino sources that are motivated by high energy gamma ray detections. One example is the Galactic center of the milky way, located $29^{\circ}$ above the horizon at the South Pole. The possible impact of a surface veto detector to astrophysical neutrino measurements with IceCube is discussed in [4].

Detector extensions to veto cosmic-ray induced signals on the surface have to be very efficient in different ways:

- A low energy threshold and high detection efficiency of a surface veto is strongly related to 
the detectable neutrino flux.

- A large duty cycle is needed for a surface veto detector to detect as many astrophysical events as possible.

- The number of detectable astrophysical events increases linearly with the azimuthal coverage of a surface array. With increasing declination the length of the active Volume (ice) increases. Thus a neutrino will more likely interact with increasing declination and produce a signal in the in-ice detector. In the vertical case, only the ice directly above the detector (about a factor of 2.5) is gained, while e.g. in the direction of the Galactic Center (declination $\theta=61^{\circ}$ ) the active volume is already about six times larger.

- The detection system has to be easy to deploy and operate.

The most obvious option for a surface veto are particle detectors which measure the Cherenkov- or scintillation light produced in an enclosed active volume [9]. First simulations based on shower parameterizations underline the importance of large detection volumes and sensitivity to the electromagnetic and the muonic component of the air shower [10]. Uncertainties from intrinsic fluctuations in the air-shower front at the surface in this case are of primary importance in determining the veto efficiency and its energy dependence.

In the following, an array of small imaging air Cherenkov telescopes is discussed as an alternative detection method which uses the atmosphere as the active volume. First we discuss an estimated air Cherenkov telescope array and the technical properties of imaging air Cherenkov telescopes needed for a surface veto detector. After that, a 61 pixel IceAct prototype telescope that has been built for the South Pole is described. In addition, we show first data that was taken with a 7 pixel IACT demonstrator at the South Pole in coincidence with IceCube.

\section{Criteria for an air Cherenkov veto array at the South Pole}

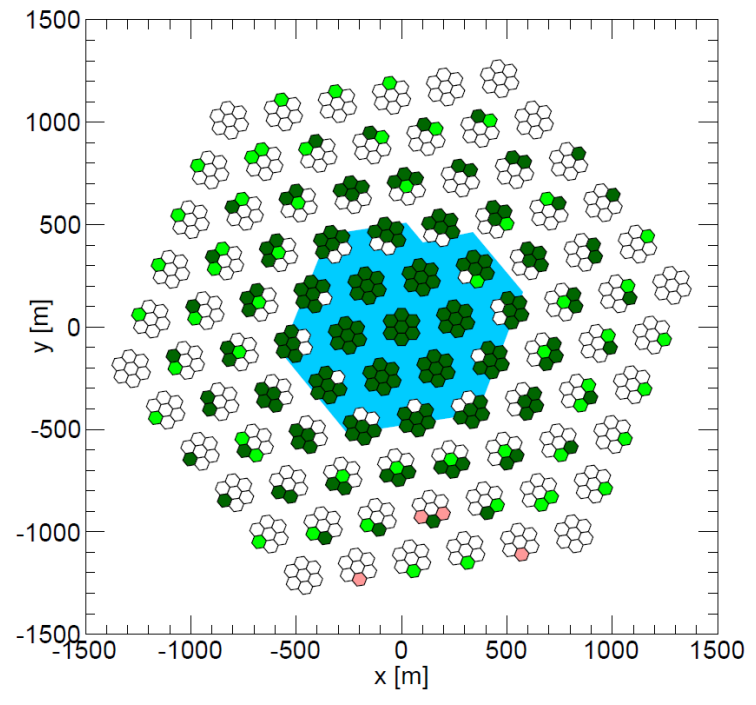

Figure 2: Add on Array for IceTop. The blue region indicates the footprint of IceTop. Each 7 connected hexagons build one station of 7 telescopes. Each telescope looks in a different direction of the sky, opening in total a field of view of $18^{\circ}$. The colors of the different telescopes (hexagons) indicate the importance of the telescope. Deep green telescopes shield $>50$ optical modules of from IceCube deep in the ice from cosmic ray signals (see the right Figure 2). The light green telescopes cover between 10 and 50 optical modules. The red telescopes cover less than 10 telescopes. The white telescopes are not needed.

In the following we will briefly discuss the motivation and required properties of possible IceAct arrays that could be operated at the South 
Pole as a surface veto for cosmic-ray air showers to detect astrophysical neutrinos. A surface detector on top of IceCube to veto air shower induced signals in the in-ice detector offers the possibility to improve the astrophysical neutrino search of IceCube in various ways. One example is the detection of well reconstructed high energy muon neutrinos [4]. Such neutrinos interacting in the ice above the instrumented volume of the deep array could be included with a calculated probability of being of astrophysical origin.

In different analyses IceCube has measured a diffuse astrophysical flux with a spectral index of $E^{-2.1}$ to $E^{-2.8}$ harder than the inclusive flux of atmospheric leptons. Thus, a veto detector needs to work increasingly more efficient the lower the energy threshold for astrophysical neutrino detection is set. Particle detector arrays on the ground suffer from a limited active detector volume. At energies where the particle density on the ground is too low, the veto efficiency will drop. As each telescope pixel of IACT's is monitoring the entire atmosphere in its field of view and the Cherenkov light emission is much more homogeneous compared to the distribution of the charged particles, the energy threshold of the IACTs is not constrained by the sparsity of the detectors. It is mainly dependent on the detection efficiency for photons, the photon background, and the photons reaching the telescope at the first place. Conventional IACTs are much to expensive to cover a large field of view. The only requirement for IACTs for the veto case is the detection of photons from certain directions within the directional uncertainty of IceCube and with a moderate probability to trigger on background photons. As a result the field of view of our pixels can be relative large above $1^{\circ}$ and the overall size of the telescope can be well below $1 \mathrm{~m}^{2}$. This drops the cost of a single telescope to well below $\$ 10000$ [11] including a DAQ that is based on Target7 [12] modules.

\subsection{IceAct Telescope application}

First simulation studies of IceAct arrays to veto cosmic ray induced background for IceCube were reported [13]. They assume the photon detection efficiency of single telescopes is comparable to those of the IceAct prototype [11]. With a 4 PE detection threshold on a night sky background taken from La Palma (accurate measurements for the South Pole need to be implemented in the future) and CORSIKA [14] simulations under South Pole conditions we find in the order of 200-300 telescopes to be sufficient to cover the most vertical $18^{\circ}$ above the IceCube in-ice detector completely (see Figure 2). Such an array of telescopes has a stable threshold at about $50 \mathrm{TeV}$ cosmic ray primary energy, well below that of IceTop (in the order of $300 \mathrm{TeV}$ ). Given that IceTop as a veto detector results in about 0.1 neutrinos/year [15] such a telescope array with a comparable field of view would be able to identify astrophysical with a neutrino/year rate dependent on the spectral index of the flux and the duty cycle of the array. Our example array covers a larger part of the IceCube in-ice detector compared to IceTop, which increases the expected number of astrophysical neutrinos further.

Another array of telescopes that is efficiently detecting cosmic ray induced background from the direction of the Galactic Center is also of particular interest as the Galactic Center is a prominent candidate for a neutrino point source motivated by the high energy gamma detection of Hess [16]. With the geographic South Pole as the frame of reference the Galactic Center rotates around IceCube with a declination of $29^{\circ}$ above the horizon. Vetoing cosmic-ray background from this region would require rings of telescopes deployed around IceCube. This type of array geometry is also under consideration and will be explored and require also several hundred telescopes. 
Finally, we note that an IceAct array of the type shown in Figure 2 would add a powerful signal component for cosmic-ray physics with IceCube. IceActs measure the air Cherenkov light emission of the electromagnetic component along the shower axis. This information is complementary to the high energy muon detection of the IceCube detector in the ice and the particle detection by IceTop on the ground [17].

\section{Development of the IceAct prototype telescope}
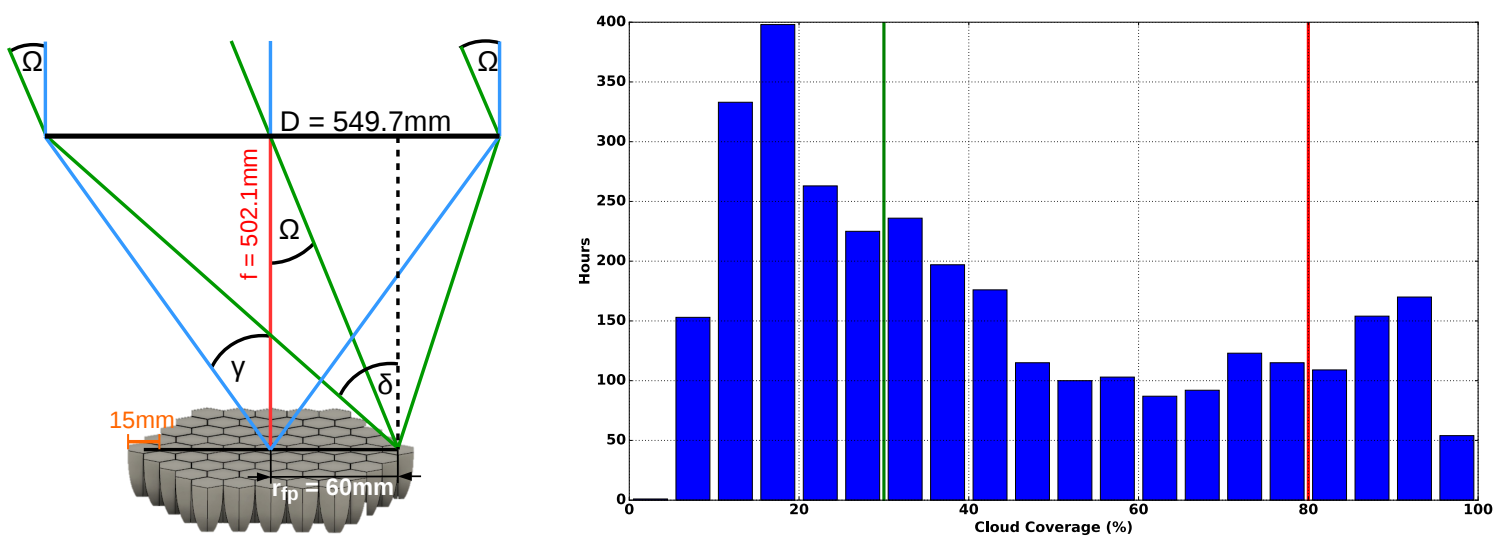

Figure 3: Left: The acceptance angle that is required for the central Winston cone and a Winston cone on the side of the camera. $\gamma$ is $28.7^{\circ}, \delta$ is $33.7^{\circ}$. Right: Duty cycle of SIPM based IACTs determined from observations with a simple $175^{\circ}$ sky camera. The plot shows the number of hours in which a certain amount of cloud coverage was found. Analyses estimate that in about $25 \%$ of the time darkness with no clouds is found in each given direction, with large uncertainties.

Efficiently running an air Cherenkov telescope at the South Pole requires special treatment of the mechanics and electronics to account for the extreme environmental properties, including low temperatures and drifting snow. Further design considerations follow from the planned use as a veto for air showers. Figure 1 shows a schematic of the current IceAct telescope design. In the following we describe the optical properties of the telescope along the signal path for incoming light. First, incoming light passes a 97\% UV-transparent glass plate that protects the entire following system from small snow crystals, dust and very cold temperatures. This is followed by a Fresnel lens with a $12^{\circ}$ field of view and a diameter of $549.7 \mathrm{~mm}$ (ORAFOL Fresnel Optics GmbH $510 \mathrm{~mm}$ SC 943 lens). A carbon cylinder sets the distance of the lens to the SiPM camera to $514 \mathrm{~mm}$ and ensures a shielding from scattered background light and snow. 61 specially designed PMMA Winston-Cones are sitting in the front of the camera. They have a hexagonal entrance window and a quadratic output window focusing the light on $6 \times 6 \mathrm{~mm}$ SensL-J SIPMs (see Figure 3 on the left). The first telescope at the South Pole had round aluminum cones and only the central 7 pixels equipped. Each single pixel of this camera has a detection efficiency of $14 \pm 3 \%$ in the region of $\pm \Delta$ at $380 \mathrm{~nm}$ wavelength including the detection efficiency of the SensL-C 6x6 mm SIPM and including the 
limited fill factor. Figure 3 motivates the reason for the chosen acceptance angle. Here $\Delta=33.7^{\circ}$ is the maximum angle in which light needs to be collected with the cones.

\subsection{Pixel Camera of the IceAct Prototype Telescope}

The central part of the IceAct prototype telescope is the 61 pixel SIPM camera. The radius $r_{f p}$ of the focal plane is $60 \mathrm{~mm}$, where the entrance window of the Winston cones is located in the focal plane of the Fresnel lens at $350 \mathrm{~nm}$ wavelength $(502.1 \mathrm{~mm})$. The left Figure 3 summarizes these numbers. The overall light detection efficiency from the direction of $0^{\circ}-\delta$ is simulated to be in the order of $32 \%$ at $380 \mathrm{~nm}$ wavelength including the detection efficiency of the SensL-C 6x6 mm SIPM and the limited fill factor.

Including the lens and the front glass the overall light detection efficiency of the telescope in its FOV is about $15 \%$ for Cerenkov light on the ground from $300-400 \mathrm{~nm}$. Wavelength and direction dependent measurements are ongoing. Measurements of the environmental properties at the South Pole for the operation of IACTs and the successful operation of IceAct prototypes at the South Pole are important to determine the potential of an extended IceAct array. One very important parameter to calculate the potential of a large IceAct array is the expected duty cycle for operation. To investigate the duty cycle of IceActs at the South Pole careful measurements of the condition of the atmosphere are necessary. A first step in this direction is the analysis of pictures that where taken with a simple $175^{\circ}$ sky camera that was installed at the South Pole from 2014-2016. During one dark period from 2015-2016 the taken data were analyzed for cloud coverage in the sky by monitoring the appearance and absence of stars. Monitoring 150 stars gives a first estimate on the overall duty cycle achievable at the South Pole. Analysis shows that for $25 \%$ of the time per year the sky in each given direction is dark and clear. Because of the large uncertainties (in both directions) of this measurement all our estimates assume that the field of view of a given telescope is dark and free of clouds or bright auroras $20 \%$ of the time. This star disappearance measurement is in agreement with LIDAR-data analyses from the South Pole [18]. A professional camera for night sky observations was installed at the South Pole in 2016. The data will be used to confirm the results of this measurement.

\subsection{Coincident Data of the IceAct Demonstrator Telescope at the South Pole}

Together with the wooden stand an IceAct demonstrator was deployed on the roof of the IceCube counting house (ICL) in 2016 to start elaborating the capabilities of IceAct to calibrate the cosmic ray flux detected by IceCube. The telescope has seven pixels for an $4^{\circ}$ field of view and can look from $45^{\circ}$ above zenith to vertical. It was operating from May 12th 2015 till August 20th 2016. Almost one month of stable data was taken in physics mode in coincidence with IceCube and IceTop. The IceAct demonstrator was autonomously triggering whenever two pixels saw a signal above threshold. In the case of a trigger in IceCube at the same time, IceCube records an IceAct trigger time stamp. In addition an independent time marker was recorded by IceAct. We also sent trigger signals to IceCube forced by two independently working $60 \mathrm{MHz}$ clocks and recorded corresponding time markers within IceAct. In case of an independent IceCube trigger the flags that are sent to IceCube are recorded. This information was used do to a time calibration between IceCube and the IceAct demonstrator. We are now able to analyze air shower events that were recorded with all three subsystems IceAct, IceTop, and IceCube. Figure 4 shows one example 


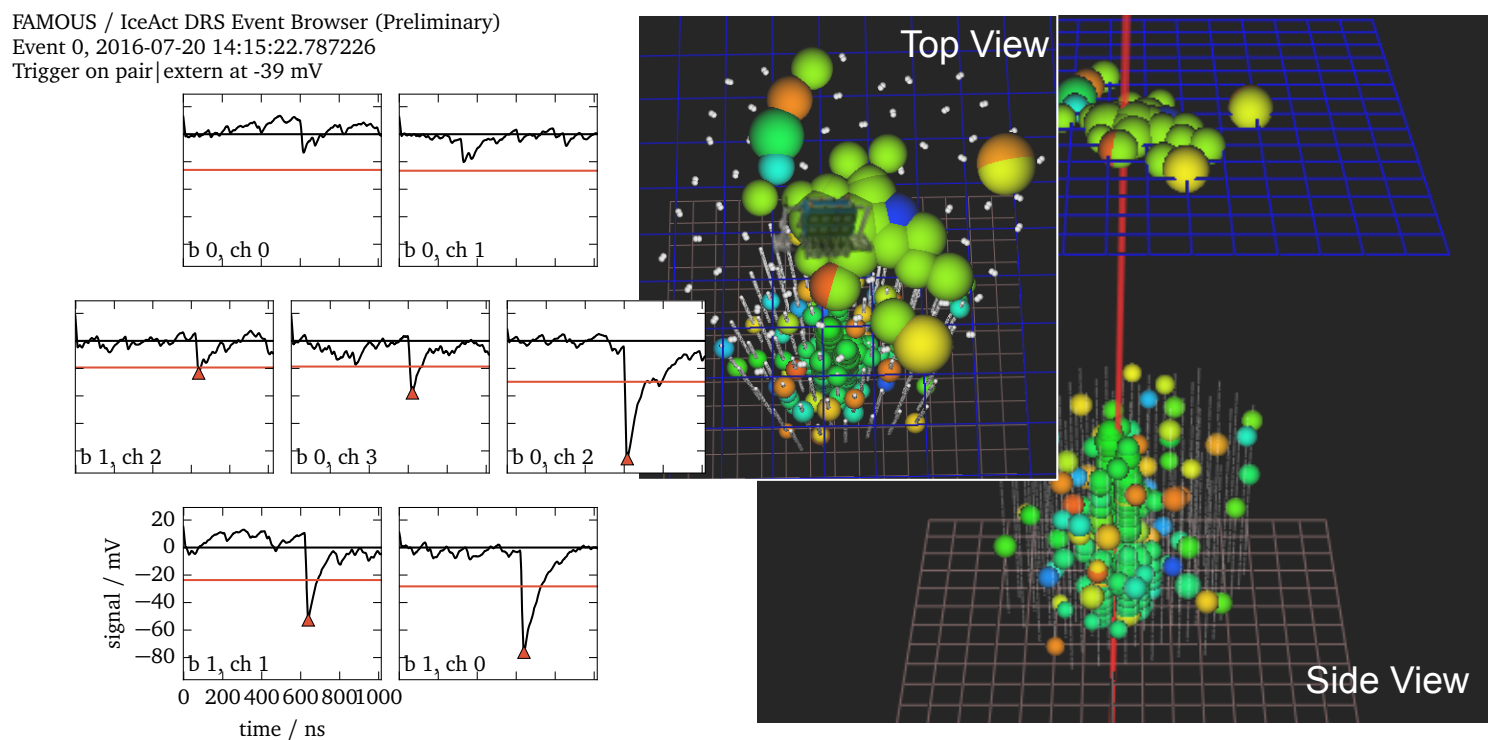

Figure 4: Example event of a coincidence of all three detector components IceTop, IceCube and IceAct.On the left one can see 5 SIPM pixels of the telescope with a clear signal above threshold. On the right is an event view of the IceTop and the IceCube light detection pattern. The color represents timing, the size of the bubble the light amount detected by the optical sensors. The time interval bins in the color code were expanded to $7 \mu$ s so that all hits in green are associated with the same event. The building (ICL) in the top view marks the position of the telescope.

event of triple coincidence. Displayed are the amount of light in IceTop on top of light signals in the deep IceCube detector. The color indicates the time of the light detection. The graphs show the corresponding SIPM pulses of the SIPM pixels from the IceAct Demonstrator. The event hit the surface close to the IceCube counting house (ICL), the location of the telescope. The ICL building is also visualized in the plot. Future goals of data analyses are to determine the energy threshold and detection efficiency as a function of the distance to the shower axis. Here the reconstruction of the air showers from IceTop and IceCube will be very helpful. This ongoing study will underline the capability to run an array of IACTs at the South Pole.

\section{Summary and Outlook}

We have described the idea of an large IceAct array to veto cosmic rays for astrophysical neutrino detection with IceCube in the southern sky. First simulations of arrays suggest that 200-300 efficiently working IceActs could be sufficient for a veto detector enhancing IceTop or covering the Galactic Center.

IceActs are small and robust telescopes that cost below $10 \mathrm{k} \$$. Being cost efficient they are a very good candidate technology to be deployed and operated in large numbers for veto detectors. A veto detector of the size of IceTop with an energy threshold one order of magnitude lower than the IceTop veto results in an additional detectable astrophysical neutrino rate of the order of one event per year dependent on the spectrum of the flux. Note that the presented IceAct array configuration 
with a covered FOV of $0^{\circ}-18^{\circ}$ declination is even larger than IceTop resulting in an additional increase of the detectable astrophysical neutrino flux.

A first IceAct demonstrator is successfully being deployed at the South Pole and is stably measuring in coincidence with IceCube and IceTop cosmic ray air shower signals.

The next IceAct prototype will be deployed in 2017 and have a 61 pixel camera to cover $0.03 \mathrm{sr}$ of the sky for cosmic ray detection and to explore duty cycle and detection efficiency of this telescope technology in situ.

\section{References}

[1] IceCube Collaboration, M. G. Aatrsen et al., JINST, 12 (2017), P03012.

[2] IceCube Collaboration, PoS (ICRC2017) 981 (these proceedings).

[3] IceCube Collaboration, PoS (ICRC2017) 997 (these proceedings).

[4] IceCube Collaboration, PoS ( ICRC2015) 1156 (2016), http://arXiv:1510.05228.

[5] IceCube Collaboration, PoS (ICRC2017) 991 (these proceedings).

[6] IceCube Collaboration, R. Abbasi et al., Nucl. Inst. Meth. A, 700 (2013) 188-220.

[7] IceCube Collaboration, PoS ( ICRC2015) 1064 (2016), https://arxiv.org/abs/1510.05223.

[8] IceCube Collaboration, M. G. Aartsen et al., ApJ 835 (2017) no.2, 151

[9] IceCube-Gen2 Collaboration, PoS ( ICRC2017) 1040 (these proceedings).

[10] IceCube Collaboration, PoS ( ICRC2015) 1070 (2016), https://arXiv:1508.06296.

[11] J. Auffenberg et al., PoS (ICRC2015) 1047 (2016), https://arxiv:1511.01680.

[12] The CTA consortium, PoS ( ICRC2015) 932 (2016), https://arXiv:1508.06296.

[13] D. Lennarz et al,AIP CP 1792, 060007 (2017), doi: 10.1063/1.4968990.

[14] D. Heck, G. Schatz, T. Thouw, J. Knapp, J. N. Capdevielle, Tech. Rep. 6019, FZKA (1998).

[15] IceCube Collaboration, PoS (ICRC2015) 1086 (2016), https://arXiv:1508.06296.

[16] HESS Collaboration, Nature 531476 (2016), http://arXiv:1603.07730v1.

[17] IceCube Collaboration, PoS (ICRC2017) 319 (these proceedings).

[18] S. Benzvi et. al., PoS (ICRC2015) 568 (2016), C15-07-30 Proceedings. 